\title{
Dual-Tunable Polarization Insensitive Electromagnetically Induced Transparency in Metamaterials
}

\author{
Renxia Ning ${ }^{1,2} \cdot$ Zhiqiang Xiao $^{3} \cdot$ Zhenhai Chen $^{1,2} \cdot$ Wei Huang $^{3}$
}

Received: 18 June 2020 / Accepted: 9 December 2020 / Published online: 20 April 2021

(c) The Author(s) 2021

\begin{abstract}
A multilayer structure of a square ring of graphene with nesting vanadium dioxide $\left(\mathrm{VO}_{2}\right)$ was investigated in this study. This structure exhibits electromagnetically induced transparency (EIT), which stems from a bright mode coupling with a dark mode. The permittivity values of graphene and $\mathrm{VO}_{2}$ can be modulated via chemical potential and temperature, respectively. The EIT effect can be tuned based on the chemical potential of graphene and temperature of $\mathrm{VO}_{2}$, resulting in a dual-tunable EIT effect. Simulation results confirmed that this dual-tunable EIT phenomenon is insensitive to polarization. These results may have potential applications in terahertz devices, such as slow light devices, switching devices, and sensors.
\end{abstract}

Keywords Electromagnetically induced transparency $\cdot$ metamaterials $\cdot$ dual-tunable $\cdot$ polarization-insensitive

\section{Introduction}

As a quantum interference effect of atomic systems, electromagnetically induced transparency (EIT) yields the characteristics of low loss, narrow bandwidth, high transparency, and strong dispersion when electromagnetic waves pass through a medium. ${ }^{1,2}$ To realize EIT effects in atomic systems, experimental conditions with very low temperature and high laser intensity are required. ${ }^{3}$ Researchers have found that design of special structural designs at room temperature can also realize similar EIT phenomena in certain materials. Such structures include waveguides, ${ }^{4,5}$ photonic crystals, ${ }^{6,7}$ and metamaterials. ${ }^{8-10}$ Using metamaterials

Renxia Ning and Zhiqiang Xiao have contributed equally to this work.

Renxia Ning

nrxxiner@hsu.edu.cn

$\triangle$ Wei Huang

eehuangw@fudan.edu.cn

1 School of Information Engineering, Huangshan University, Huangshan 245041, China

2 Engineering Technology Research Center of Intelligent Microsystems, Huangshan 245041, AnHui Province, China

3 State Key Laboratory of ASIC and System, Shanghai Institute of Intelligent Electronics and Systems, School of Microelectronics, Fudan University, Shanghai 200433, China to produce the EIT effect not only can yield electromagnetic characteristics similar to those of an atomic system, but also avoid strict experimental requirements. Therefore, such materials have been widely studied in the field of electromagnetic technology. ${ }^{11}$ The EIT effects of metamaterials have wide application prospects for optical storage, ${ }^{12}$ slow light technology, ${ }^{13}$ and high-sensitivity sensors. ${ }^{14,15}$

The main advantage of metamaterials is that they are able to provide properties that are not possessed by conventional materials based on structural changes. ${ }^{16}$ However, this can also be a disadvantage. This is because once a final structure is developed, results can no longer be tuned. Therefore, achieving tunability of results has become a major research focus. ${ }^{17-20}$

There are many types of active or tunable materials in nature that have been incorporated into metamaterials, such as ferroelectrics, ${ }^{21}$ liquid crystals, ${ }^{22,23}$ semiconductors, ${ }^{24,25}$ two-dimensional materials, ${ }^{26-29}$ and phase change materials. ${ }^{30,31}$ Xiao et al. proposed a graphene-based metasurface structure that exhibits a tunable EIT response under mid-infrared frequencies. This result demonstrated that EIT responses can be dynamically shifted in the frequency domain by adjusting the Fermi level of graphene. ${ }^{26}$ Xiao et al. investigated a planar terahertz EIT metamaterial to realize the maximum modulation depths in two perpendicular polarization directions $(83.54 \%$ and $94.39 \%$, respectively) by tuning Fermi energy. ${ }^{32} \mathrm{Xu}$ et al. proposed a simple EIT structure composed of a cutting wire and two circular 
split rings. The passive modulation of EIT metamaterials was realized based on the tunable design of this structure. ${ }^{33}$ Liu et al. designed a periodic structure with a special shape and achieved an obvious plasmon-induced transparency phenomenon based on monolayer black phosphorus (BP). This tunable plasmon-induced transparency phenomenon can be obtained by changing the carrier density to adjust resonant intensity and frequency. ${ }^{34}$

As mentioned above, a single-tunable EIT response can be achieved using graphene, $\mathrm{VO}_{2}$, $\mathrm{BP}$, etc. Recently, some researchers have studied dual-tunable metamaterials to achieve specific functions. Liu et al. investigated dual-tunable EIT based on a graphene metamaterial. The transparency window was altered by mechanically adjusting the incidence angle and Fermi energy by modifying the gate bias. ${ }^{35}$ Mao et al. designed a dielectric metasurface consisting of a $\mathrm{VO}_{2}$ and graphene array to fabricate a broadband absorber in the mid-infrared range. The maximum absorption reached as high as $99.41-99.89 \% .{ }^{36}$ Wang et al. proposed a dual-tunable switchable broadband $\mathrm{THz}$ metamaterial absorber based on a hybrid $\mathrm{VO}_{2}$ and graphene structure. The peak absorption of this device can be tuned from $26 \%$ to $99.2 \%$ and from $9 \%$ to $99.2 \%$ by modifying the Fermi energy of the graphene and conductivity of the $\mathrm{VO}_{2}$, respectively. ${ }^{37}$

Dual-tuning can be achieved by combining two tunable materials with different characteristics. However, there have been few reports on dual-tunable EIT effects. A single and dual-band EIT effect was investigated in a microwave metamaterial, which exhibited high transmission. ${ }^{16}$ A polarization insensitive double-band EIT phenomenon is studied experimentally in the terahertz band. ${ }^{10}$ These results can be applied in the fields on filters and sensors. Among these few reports, dual-tunable polarization-insensitive EIT is the most common goal.

In this paper, a unit cell with a tri-layer structure is proposed based on the time-domain finite-integral method. The proposed structure is composed of a square ring of graphene with nested $\mathrm{VO}_{2}$ in an asymmetric $T$ shape, with a magnesium fluoride $\left(\mathrm{MgF}_{2}\right)$ and silicon dioxide $\left(\mathrm{SiO}_{2}\right)$ substrate.
$\mathrm{VO}_{2}$ is a temperature-tunable phase change material that has metallic properties at high temperatures and insulating properties at room temperature. Therefore, the proposed structure can achieve multiple functions by adjusting temperature and voltage independently. Polarization-insensitive EIT can be realized based on numerical calculations. Simulation results indicate that the proposed structure could be applied to $\mathrm{THz}$ sensors, slow light devices, and switching devices.

\section{Design and Theory}

The electromagnetic properties of a graphene sheet are described in terms of the surface conductivity $\sigma$, which accounts for both inter-band and intra-band transitions based on the Kubo model of conductivity. This conductivity is defined as ${ }^{38,39}$

$$
\begin{aligned}
\sigma= & \frac{i e^{2} k_{B} T}{\pi \hbar^{2}(\omega+i / \tau)}\left(\frac{\mu_{c}}{k_{B} T}+2 \ln \left(e^{-\frac{\mu_{c}}{k_{B} T}}+1\right)\right) \\
& +\frac{i e^{2}}{4 \pi \hbar} \ln \left|\frac{2 \mu_{c}-\hbar(\omega+i / \tau)}{2 \mu_{c}+\hbar(\omega+i / \tau)}\right|,
\end{aligned}
$$

where $\omega$ is the radian frequency, $\hbar$ is the reduced Planck constant, $\kappa_{\mathrm{B}}$ is the Boltzman constant, $e$ is the charge of an electron, $T$ is the temperature, $\mu_{\mathrm{c}}$ is the chemical potential, and $\tau$ is the electron-phonon relaxation time.

We assume that the electronic band structure of a graphene sheet is not affected by neighboring structures, so the effective permittivity $\varepsilon_{\mathrm{G}}$ of graphene can be written as ${ }^{40}$

$\varepsilon_{G}=1+i \frac{\sigma}{h_{3} \omega \varepsilon_{0}}$,

where $h_{3}$ is the thickness of the graphene sheet and $\varepsilon_{0}$ is the permittivity in a vacuum.

The spectra of graphene permittivity are plotted in Fig. 1 for $\tau=1 \mathrm{ps}$. An increase in the chemical potential $\mu_{\mathrm{c}}$ of graphene can suppress the real part of $\varepsilon_{\mathrm{G}}$ and enhance the

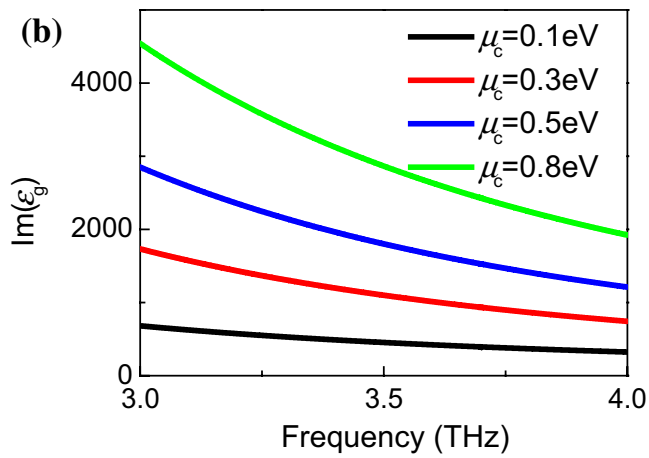

Fig. 1 (a) Real and (b) imaginary part of the permittivity of graphene with different values of $\mu_{\mathrm{c}}$ of $h_{3}=1 \mathrm{~nm}$. 
imaginary part of $\varepsilon_{\mathrm{G}}$. This indicates that the permittivity of graphene can be tuned by altering its chemical potential $\mu_{\mathrm{c}}$.

Furthermore, the permittivity of graphene remains unchanged with changing temperature. ${ }^{41,42}$

As a phase change material, the phase transition temperature of $\mathrm{VO}_{2}$ is approximately $340 \mathrm{~K}$. It can be considered to exhibit an insulating state prior to phase transition. Within the $\mathrm{THz}$ range, the relative permittivity of $\mathrm{VO}_{2}$ can be expressed by the Drude model ${ }^{31}$ as

$\varepsilon_{\mathrm{VO}_{2}}=\varepsilon_{\infty}-\frac{\omega_{p}^{2}\left(\sigma_{\mathrm{VO}_{2}}\right)}{\omega(\omega+i \gamma)}$,

where $\varepsilon_{\infty}=12$. Experimental results have demonstrated that the conductivity $\sigma_{\mathrm{vo}_{2}}$ of $\mathrm{VO}_{2}$ varies from 10 to $2 \times 10^{5} \mathrm{~S} / \mathrm{m},{ }^{43}$ where $\gamma=5.75 \times 10^{13} \mathrm{~s}^{-1}$ and $\omega_{p}\left(\sigma_{\mathrm{vo}_{2}}\right)=\omega_{p}\left(\sigma_{0}\right)\left(\sigma_{\mathrm{vo}_{2}} / \sigma_{0}\right)^{0.5} \cdot{ }^{37}$

We propose a tri-layer unit cell consisting of graphene with nested $\mathrm{VO}_{2}$ on a $\mathrm{MgF}_{2}$ and $\mathrm{SiO}_{2}$ substrate. The $\mathrm{VO}_{2}$ layer is hollowed out in an asymmetric $T$ shape. This unit cell is depicted in Fig. 2. The finite-integral time-domain method was utilized to calculate the normal incident angle $\left(\theta=0^{\circ}\right)$ of the electromagnetic waves of periodic boundary conditions for the $x$ and $y$ axes and of open spaces for the $z$ axis. The electric $(\mathrm{E})$ and the magnetic $(\mathrm{H})$ fields were polarized along the $y$ - and $x$-directions, respectively, while the wave propagation $(\mathrm{k})$ was normal to the structure. The dielectric permittivity values of $\mathrm{SiO}_{2}$ and $\mathrm{MgF}_{2}$ are 3.9 and 1.9 , respectively. We assume that electromagnetic waves propagate along the $\mathrm{z}$ axis. The thicknesses of the layers in the proposed structure are denoted as $h_{1}, h_{2}$, and $h_{3}$ from bottom to top. The geometry of the unit cell is defined by

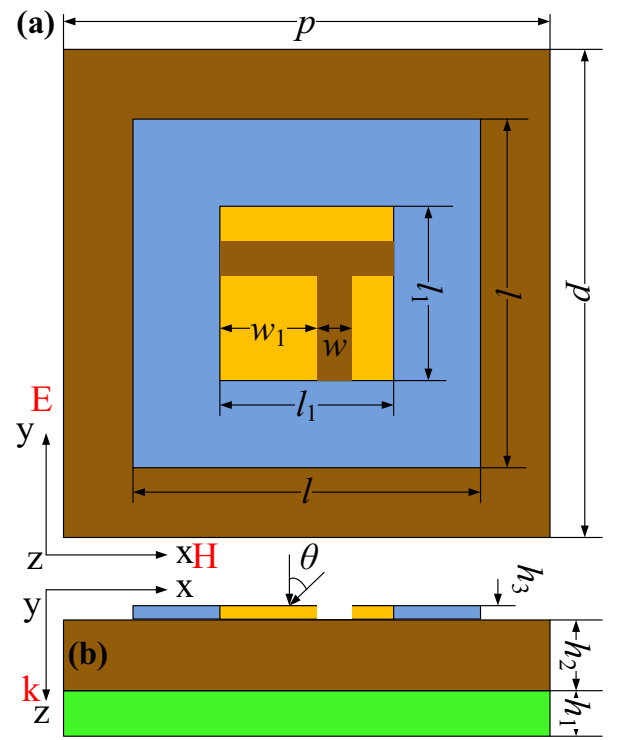

Fig. 2 (a) Front view and (b) side view of the proposed unit cell (trilayer metamaterial). $p=120 \mu \mathrm{m}, h_{1}=1 \mu \mathrm{m}, h_{2}=2 \mu \mathrm{m}, h_{3}=0.01 \mu \mathrm{m}, l=80 \mu \mathrm{m}$, $l_{1}=10 \mu \mathrm{m}, w=2.5 \mu \mathrm{m}$, and $w_{1}=5 \mu \mathrm{m}$.

Figure 3 presents the transmission spectra of structures with (a) $\mathrm{VO}_{2}$ and (b) graphene, and (c) the integrated multilayer structure. The resonant frequency is generated at $3.388 \mathrm{THz}$ in Fig. $3 \mathrm{a}$ (only $\mathrm{VO}_{2}$ and a dielectric substrate). It is worth noting that the transmission level is greater than 0.92 at the resonant frequency. The dual-resonant frequencies occur at $3.421 \mathrm{THz}$ and $3.571 \mathrm{THz}$ in Fig. $3 \mathrm{~b}$ (only graphene and a dielectric substrate). Compared to the structure with $\mathrm{VO}_{2}$, the transmission of the structure with graphene is very small. The transmission of the integrated multilayer structure containing $\mathrm{VO}_{2}$ and graphene differs from those of the separate $\mathrm{VO}_{2}$ and graphene structures. The EIT effect can be observed in the lowfrequency band. According to previous reports, the judgment of bright modes and dark modes can be performed based on the $Q$-factor, which is defined as $Q=f_{0} / \Delta f$, where $f_{0}$ is the resonant frequency of the transmission peak and
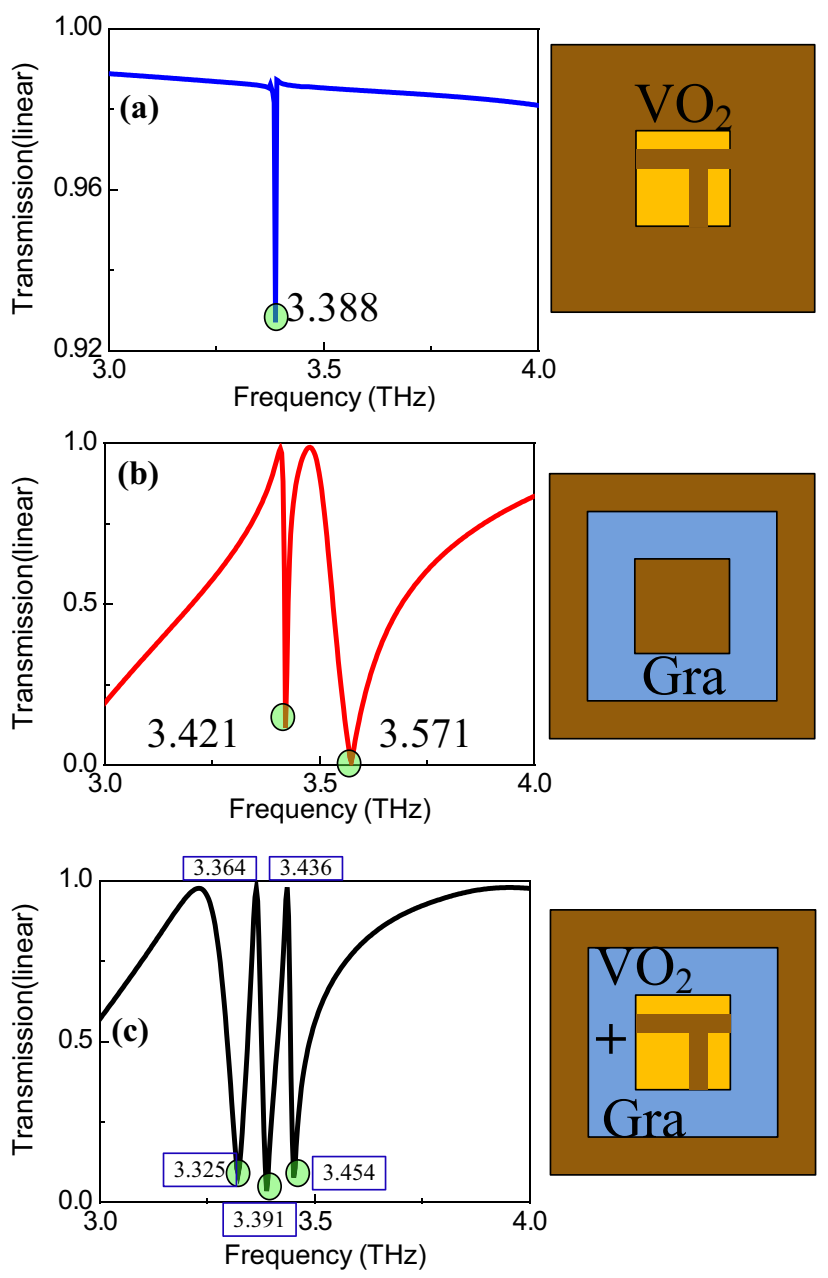

Fig. 3 Transmission spectra of (a) $\mathrm{VO}_{2}$, (b) graphene, and (c) the integrated multilayer structure. 
$\Delta f$ is the full width at half maximum of the transmission peak. ${ }^{44}$ A bright mode is observed in the structure of $\mathrm{VO}_{2}$ at low $Q$-factor values and a dark mode is observed in the structure of graphene with high $Q$-factor values (sharper transmission valley).

To help us understand the physical mechanisms behind the observed EIT effect, we visualize the peak distributions of the electric field of the proposed structure in Fig. 4. One can see that the distributions of the electric field at 3.325, 3.391, and 3.454 THz (Fig. 3a, c, and e, respectively) are particularly intense. In Fig. 4a, one can see that at the resonant frequency of $3.325 \mathrm{THz}$, the electric field is concentrated in the y direction at the interface between the $T$-type $\mathrm{VO}_{2}$ and graphene. This indicates that $T$-type $\mathrm{VO}_{2}$ can respond to incident electromagnetic waves at this frequency, so it exhibits a bright mode. In Fig. 4b, energy is coupled between the junction of the $T$-type $\mathrm{VO}_{2}$ and graphene, and the outer boundary of the square ringshaped graphene. In this case, the graphene exhibits a dark mode and the $\mathrm{VO}_{2}$ is coupled in an open mode. In Fig. 4c, at the transmission valley point of $3.391 \mathrm{THz}$, the direction of the electric field at the outer boundary of the square graphene ring changes. Here, graphene in a dark mode is coupled with $\mathrm{VO}_{2}$ in a bright mode, resulting in a new resonant frequency point. A similar phenomenon also occurs in Fig. 4e, but the direction of the electric field also changes.

\section{Results and Discussion}

As mentioned previously, the effective permittivity $\varepsilon_{\mathrm{G}}$ of graphene can be regulated by altering its chemical potential $\mu_{\mathrm{c}}$. However, altering the temperature $T$ has no effect. In contrast, the permittivity $\varepsilon_{\mathrm{VO} 2}$ of $\mathrm{VO}_{2}$ can be adjusted by altering $T$. This means that the dielectric constants of graphene and $\mathrm{VO}_{2}$ can be adjusted by altering voltage and temperature, respectively. Therefore, a structure combining these two materials can act as a dual-tunable metamaterial.

Regarding this dual-tunable EIT effect, one can see that transmission varies with the chemical potential $\mu_{\mathrm{c}}$ of graphene in Fig. 5a. A blue shift can be observed with an increasing value of $\mu_{\mathrm{c}}$ at $T=300 \mathrm{~K}$. This is because the real part of the permittivity of graphene decreases with increasing $\mu_{\mathrm{c}}$, while the imaginary part increases with increasing $\mu_{\mathrm{c}}$. Figure $5 \mathrm{~b}$ presents the transmission with different values of $\mu_{\mathrm{c}}$ at $T=340 \mathrm{~K}$. One can see that the transmission window exhibits a blue shift as $\mu_{\mathrm{c}}$ increases from $0.1 \mathrm{eV}$ to $0.3 \mathrm{eV}$. As $\mu_{\mathrm{c}}$ continues to increase to $0.8 \mathrm{eV}$, the transmission window becomes invariant. This is because when the chemical potential of graphene changes, the conductivity of graphene also changes, meaning its response to electromagnetic waves also changes.

We also explored the influence of temperature $T$ on the transmission window. Figure 6 presents the changes in the transmission window at different temperatures for

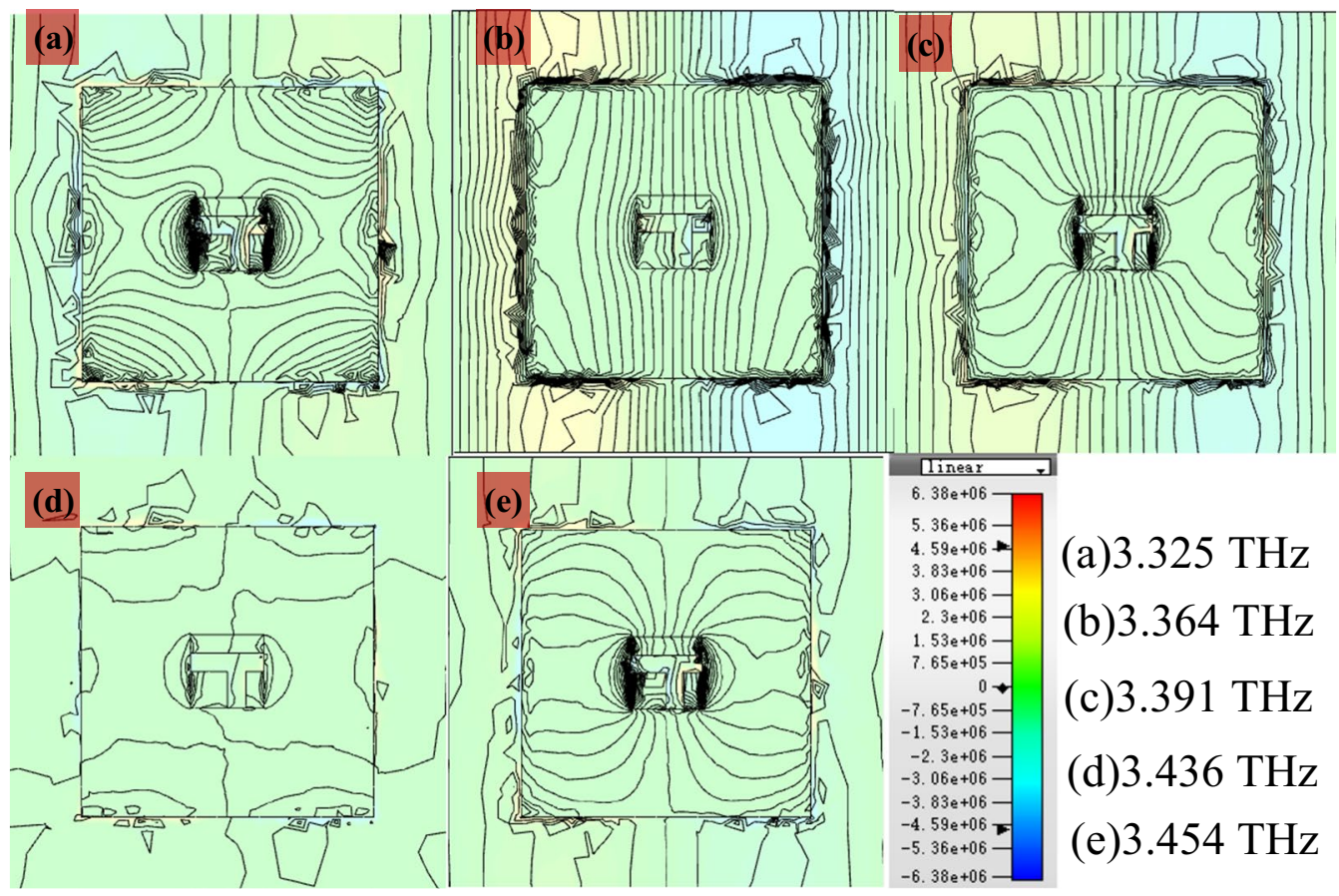

Fig. 4 Distributions of the electric field of the proposed structure at various frequencies of (a) 3.325, (b) 3.364, (c) 3.391, (d) 3.436, and (e) $3.454 \mathrm{THz}$. 
(a)

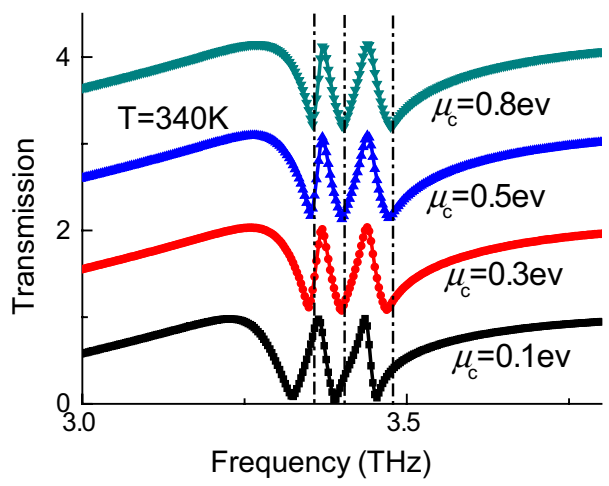

(b)

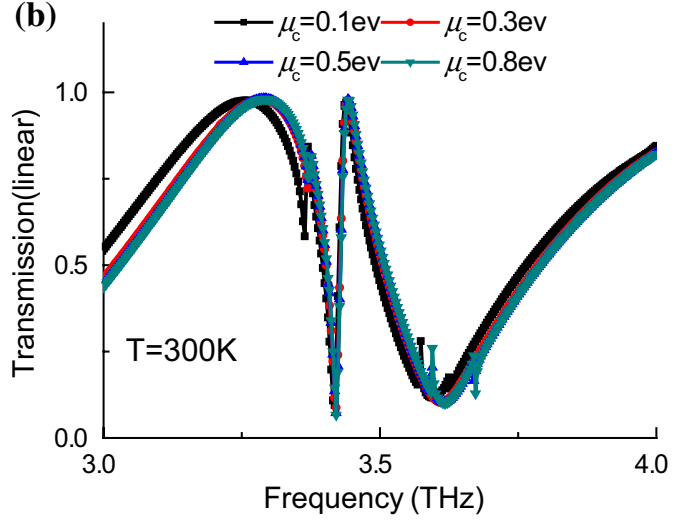

Fig. 5 Transmission windows for different $\mu_{\mathrm{c}}$ at (a) $T=340 \mathrm{~K}$ and (b) $T=300 \mathrm{~K}$.

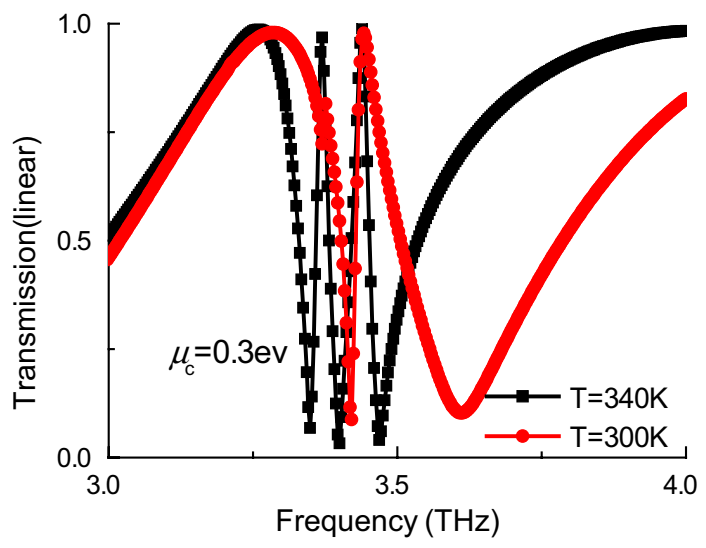

Fig. 6 Transmission windows of the proposed structure for different $T$ values at $\mu_{\mathrm{c}}=0.3 \mathrm{eV}$.

$\mu_{\mathrm{c}}=0.3 \mathrm{eV}$. One can clearly observe the effect of temperature on the EIT window. $\mathrm{VO}_{2}$ exhibits metal characteristics and can couple with graphene in a dark mode when the temperature $T$ is $340 \mathrm{~K}$. However, $\mathrm{VO}_{2}$ acts as an insulator and cannot be coupled with graphene when the temperature drops to $300 \mathrm{~K}$. This is because the transition of $\mathrm{VO}_{2}$ between an insulator and metal occurs at approximately $340 \mathrm{~K}$. With an increase in temperature, the lattice structure of $\mathrm{VO}_{2}$ transforms from monoclinic to tetragonal, so its conductivity increases by several orders of magnitude. In other words it transitions from an insulating state to a metal state.

In addition to being adjustable based on different parameters, this structure also provides polarization insensitivity. Figure 7 presents the variation in the transmission spectra with the polarization angle $\varphi$. One can see that the transmission window remains the same as $\varphi$ changes from $0^{\circ}$ to $90^{\circ}$. Polarization-insensitive EIT can be realized based on the $x / y$-axis symmetry of graphene as a bright mode and $x / y$-axis asymmetry of $\mathrm{VO}_{2}$ as a dark mode.

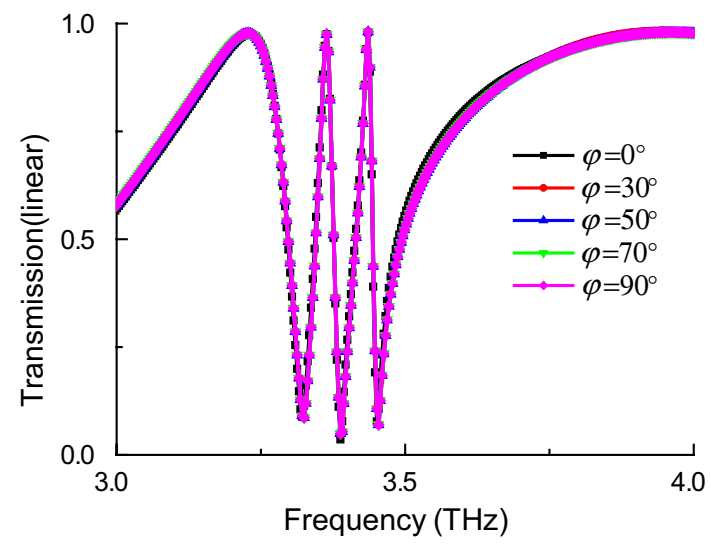

Fig. 7 Transmission spectra of the multilayer structure for various polarization angles $\varphi$.

Based on the narrow EIT line width, THz sensors are one potential application of the EIT effects of the proposed metamaterial structure. In this context, we analyzed the proposed structure with different background permittivity values. Figure 8 presents the transmission spectra for different background permittivity values varying from 1.0 to 1.6. One can clearly see a red shift in the EIT window with increasing background permittivity. The EIT peak position keeps moving to lower frequencies until the background permittivity reaches 1.6.

\section{Conclusion}

In this paper, a tri-layer graphene square ring structure with nested $\mathrm{VO}_{2}$ was proposed to realize and manipulate the EIT effect. A dual-tunable EIT effect was obtained because graphene and $\mathrm{VO}_{2}$ can be regulated by altering their chemical potential and temperature, respectively. The square graphene ring provides a bright mode that is coupled with the $\mathrm{VO}_{2}$ and 

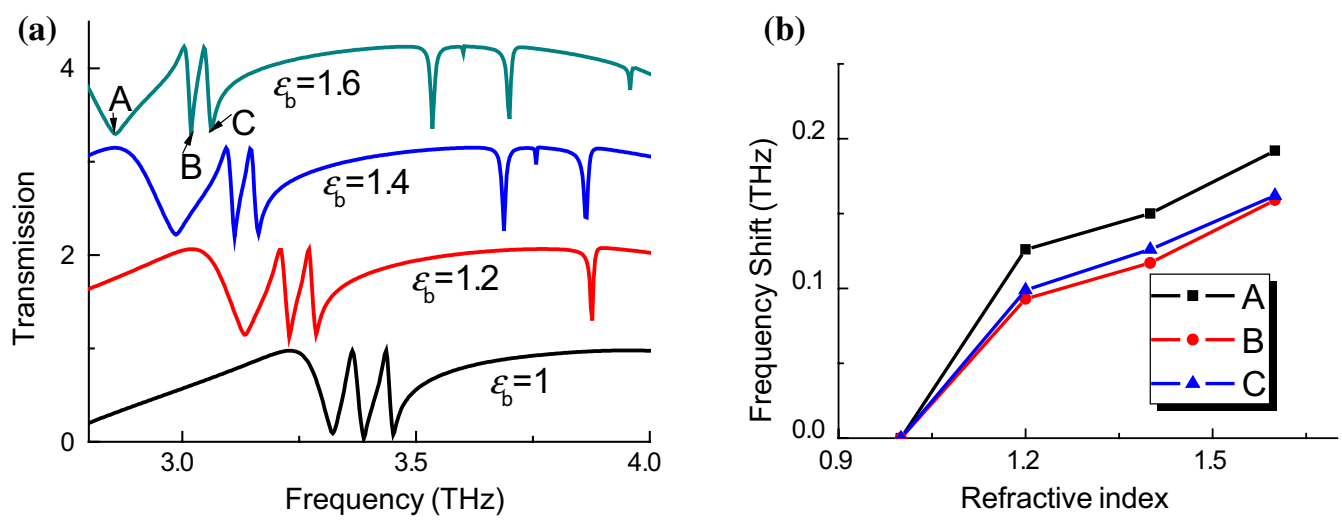

Fig. 8 (a) Variations in transmission spectra and (b) frequency shift of the transmission dips with different background permittivity values.

substrate layers, which provide a dark mode. Furthermore, polarization-insensitive EIT can also be manipulated. Based on these results, potential sensor applications were highlighted, which should provide useful guidance for additional future applications.

Funding This study was founded by Natural Science Research Project of Anhui Province Education Department (Grant No. KJ2018A0407), Major Science and Technology Projects in Anhui Province (18030901006)

Conflict of interest The Authors of Renxia Ning and Zhenhai Chen have received research grants from Huangshan University. Zhiqiang Xiao and Wei Huang has received research grants from Fudan University.

Open Access This article is licensed under a Creative Commons Attribution 4.0 International License, which permits use, sharing, adaptation, distribution and reproduction in any medium or format, as long as you give appropriate credit to the original author(s) and the source, provide a link to the Creative Commons licence, and indicate if changes were made. The images or other third party material in this article are included in the article's Creative Commons licence, unless indicated otherwise in a credit line to the material. If material is not included in the article's Creative Commons licence and your intended use is not permitted by statutory regulation or exceeds the permitted use, you will need to obtain permission directly from the copyright holder. To view a copy of this licence, visit http://creativecommons.org/licenses/by/4.0/.

\section{References}

1. K.-J. Boller, A. Imamoğlu, and S.E. Harris, Phys. Rev. Lett. 66, 2593 (1991).

2. S.E. Harris, Electromagnetically induced transparency, in Quantum Electronics and Laser Science Conference, QTuB1 (1997).

3. H. Xia, S.J. Sharpe, A.J. Merriam, and S.E. Harris, Phys. Rev. A 56, 315 (1997).

4. C. Zhao and C. Zhang, Pramana 92, 13 (2019).

5. F. Bagci and B. Akaoglu, Phys. Lett. A 383, 126000 (2019).

6. X. Yang, M. Yu, D.-L. Kwong, and C.W. Wong, Phys. Rev. Lett. 102,173902 (2009).
7. F. Jiang, C.-S. Deng, Q. Lin, and L.-L. Wang, Opt. Express 27, 32122 (2019).

8. W. Cai, Y. Fan, X. Huang, Q. Fu, R. Yang, W. Zhu, and F. Zhang, Phys. Rev. A 100, 053804 (2019).

9. S. Hu, D. Liu, and H. Yang, J. Phys. D Appl. Phys. 52, 175305 (2019).

10. R. Sarkar, K.M. Devi, D. Ghindani, S.S. Prabhu, D.R. Chowdhury, and G. Kumar, J. Opt. 22, 035105 (2020).

11. X. Yan, M. Yang, Z. Zhang, L. Liang, D. Wei, M. Wang, M. Zhang, T. Wang, L. Liu, and J. Xie, Biosens. Bioelectron. 126, 485 (2019).

12. S. Zhang, S. Zhou, M.M. Loy, G.K.L. Wong, and S. Du, Opt. Lett. 36, 4530 (2011).

13. A.H. Safavi-Naeini, T.M. Alegre, J. Chan, M. Eichenfield, M. Winger, Q. Lin, J.T. Hill, D.E. Chang, and O. Painter, Nature 472, 69 (2011).

14. N. Liu, T. Weiss, M. Mesch, L. Langguth, U. Eigenthaler, M. Hirscher, C. Sonnichsen, and H. Giessen, Nano Lett. 10, 1103 (2010).

15. R. Li, X.-K. Kong, S.-B. Liu, Z.-M. Liu, and Y.-M. Li, Phys. Lett. A 383, 125947 (2019).

16. O. Demirkap, F. Bagci, A. Yilmaz, and B. Akaoglu, Adv. Electromag. 8, 63 (2019).

17. S. Xiao, T. Wang, T. Liu, X. Yan, Z. Li, and C. Xu, Carbon 126, $271(2018)$.

18. X. Li, R. Xie, W. Li, Z. Li, E. Gu, L. Niu, and S. Guo, Superlattices Microstruct. 128, 342 (2019).

19. C. Shu and J.-S. Mei, Opt. Commun. 439, 16 (2019)

20. T. Wang, M. Cao, Y. Zhang, and H. Zhang, Opt. Mater. Express 9, 1562 (2019).

21. T.H. Hand and S.A. Cummer, Frequency Tunable Electromagnetic Metamaterial Using Ferroelectric Loaded Split Rings (College Park: American Institute of Physics, 2008).

22. D. Shrekenhamer, W.-C. Chen, and W.J. Padilla, Phys. Rev. Lett. 110,177403 (2013).

23. J. Yang, P. Wang, T. Shi, S. Gao, H. Lu, Z. Yin, W. Lai, and G. Deng, Opt. Express 27, 27039 (2019).

24. A. Keshavarz and Z. Vafapour, JOSA B 36, 35 (2019).

25. S. Liu, I. Brener, and M.B. Sinclair, Active Optical Device Enabled by Dielectric Metamaterials (New York: Google Patents, 2019).

26. B. Xiao, S. Tong, A. Fyffe, and Z. Shi, Opt. Express 28, 4048 (2020).

27. C. Qiu, J. Wu, R. Zhu, L. Shen, and B. Zheng, Opt. Commun. 451, 226 (2019). 
28. J. Ji, S. Zhou, W. Wang, F. Ling, and J. Yao, Nanoscale 11, 9429 (2019).

29. S.X. Xia, X. Zhai, L.L. Wang, B. Sun, J.Q. Liu, and S.C. Wen, Opt. Express 24, 17886 (2016).

30. J. Zhang, S. Xu, L. Huang, Z. Xu, J. Huang, L. Lei, and P. Xu, Dynamic broadband metamaterial absorber in $\mathrm{VO}_{2}$, in Asia Communications and Photonics Conference, M4A. 249 (2019).

31. J. Li, Y. Zhang, J. Li, J. Li, Y. Yang, J. Huang, C. Ma, Z. Ma, Z. Zhang, L. Liang, and J. Yao, Opt. Commun. 458, 124744 (2020).

32. M. Chen, Z. Xiao, X. Lu, F. Lv, and Y. Zhou, Carbon 159, 273 (2020).

33. Y. Xu, X. Wang, X. Chen, and L. Zhang, J. Appl. Phys. 127, $034501(2020)$.

34. C. Liu, H. Li, H. Xu, M. Zhao, C. Xiong, B. Zhang, and K. Wu, J. Phys. D Appl. Phys. 52, 405203 (2019).

35. C. Liu, P. Liu, C. Yang, Y. Lin, and H. Liu, Carbon 142, 354 (2019).

36. M. Mao, Y. Liang, R. Liang, L. Zhao, N. Xu, J. Guo, F. Wang, H. Meng, H. Liu, and Z. Wei, Nanomaterials 9, 1101 (2019).
37. T. Wang, Y. Zhang, H. Zhang, and M. Cao, Opt. Mater. Express 10, 369 (2020).

38. S.A. Mikhailov and K. Ziegler, Phys. Rev. Lett. 99, 016803 (2007).

39. A.C. Ferrari, J. Meyer, V. Scardaci, C. Casiraghi, M. Lazzeri, F. Mauri, S. Piscanec, D. Jiang, K. Novoselov, and S. Roth, Phys. Rev. Lett. 97, 187401 (2006).

40. A. Vakil and N. Engheta, Science 332, 1291 (2011).

41. R. Ning, S. Liu, H. Zhang, X. Kong, B. Bian, and J. Bao, J. Opt. 16, 125108 (2014).

42. L. Falkovsky, Optical properties of graphene. in Journal of Physics: Conference Series, p. 012004 (2008).

43. P.U. Jepsen, B.M. Fischer, A. Thoman, H. Helm, J.Y. Suh, R. Lopez, and R.F. Haglund, Phys. Rev. B 74, 205103 (2006).

44. C. Liu, P. Liu, L. Bian, Q. Zhou, G. Li, and H. Liu, Opt. Commun. 410, 17 (2018)

Publisher's Note Springer Nature remains neutral with regard to jurisdictional claims in published maps and institutional affiliations. 